\title{
La autoevaluación. Una propuesta formativa e innovadora
}

\section{The self-evaluation. A formative and innovative proposal}

\author{
María Amparo Calatayud Salom \\ Universidad de Valencia (UV), España
}

\section{Resumen}

El presente artículo propone la necesidad de potenciar la autoevaluación entre el profesorado iberoamericano como estrategia de mejora de las prácticas docentes y como recurso de formación. En estos momentos, más si cabe, es necesaria la formación en autoevaluación puesto que ésta facilita y beneficia el desarrollo y el crecimiento tanto personal como profesional. En esta investigación se presentan tanto los supuestos teóricos y las implicaciones metodológicas que alimentan la implementación de prácticas autoevaluativas, así como también, se exponen los resultados cualitativos obtenidos con una muestra de 150 profesores y profesoras de la escuela pública española. Dichos resultados han ayudado a desvelar las dificultades más arraigadas para la emergencia de la autoevaluación entre el profesorado de Educación Primaria. Desde la mirada del profesorado podemos comprender cuáles son sus dificultades para así potenciar una formación en autoevaluación que incida en que ésta sea entendida como una necesidad y una exigencia en la profesión docente hoy.

Palabras clave: Autoevaluación; formación; mejora; desarrollo profesional.

\begin{abstract}
This article proposes the need to promote self-evaluation among teachers as a strategy to improve teaching practices and teacher training resources. In these moments, more if possible, training in self-evaluation is necessary since it facilitates and benefits both personal and professional development and growth. This research presents both the theoretical assumptions and the methodological implications that feed the implementation of self-evaluative practices, as well as the qualitative results obtained with a sample of 150 teachers from the Spanish public school. These results have helped to reveal the most ingrained difficulties for the emergence of self-evaluation among nursery and primary teachers. From the teachers' point of view, we can understand what their difficulties are in order to strengthen a self-evaluation training that influences its being understood as a need and a requirement in the teaching profession today.
\end{abstract}

Keywords: Self-evaluation; training; improvement; professional development.

\section{A MODO DE INTRODUCCIÓN}

La formación en procesos autoevaluativos es, en estos momentos, de suma importancia para la mejora de las prácticas educativas. El escenario educativo incierto y cambiante cada vez está más necesitado de la competencia autoevaluativa. Competencia fundamental para el desarrollo y mejora tanto profesional como personal. Como afirman Cano (2005), Coll (2007), Charlier (2005) y Perrenoud (2007), si estamos frente a nuevas prácticas educativas, se requieren en estos momentos nuevas competencias docentes para este nuevo escenario educativo, 
para este nuevo modelo de docencia, por lo que estamos llamados a caminar hacia la construcción de un nuevo oficio de enseñar. Desde la construcción de este nuevo modelo, la competencia autoevaluativa ocupa un peso determinante para la formación docente.

El profesor, como profesional autónomo y responsable que es, necesita conocer por medio de la autoevaluación cual es el nivel de aprendizaje con el que empiezan los estudiantes-fase de diagnóstico inicial- y también su evolución a través del proceso didáctico, cuáles son sus dificultades y las causas que las originan; ya que solamente de esta manera podrá tratar de adaptar convenientemente su actuación sobre ellos. Además, el docente ha de conocer, también, cual es la eficiencia de su actuación, el papel de los recursos y de la metodología que utiliza, el grado de satisfacción de la programación prevista, etc.

Para el desarrollo de la competencia autoevaluativa se puede contar con la colaboración de otros colegas que asesoren y ayuden a programar líneas y procedimientos para superar las dificultades, para reforzar los aspectos positivos, para diagnosticar las necesidades de formación del docente, etc. ya sea mediante la recogida de cuestionarios cumplimentados por los propios estudiantes en donde emiten sus opiniones sobre los múltiples aspectos de la acción docente, etc.

Sin duda alguna, una de las características que definen a un buen profesional es la capacidad de reflexionar sobre su propia práctica y por esta razón, es necesario introducir estrategias que permitan su desarrollo (Guzmán y Marín, 2011; Cano, 2008; Bozu y Canto, 2009). De ahí, la necesidad de apostar por la emergencia de una competencia profesional que incida en la autoevaluación. Como argumenta Calatayud (2007), para "aprender a mejorar su práctica profesional, el docente ha de contrastar sus teorías previas con las evidencias de una reflexión rigurosa sobre su quehacer. La reflexión sobre su propia acción es un componente esencial del proceso de aprendizaje permanente que constituye el eje de la formación profesional" (pág. 54).

\section{LA AUTOEVALUACIÓN COMO ESTRATEGIA DE FORMACIÓN Y DE EVALUACIÓN DE LA PRÁCTICA DOCENTE}

Aprender a educar en un mundo complejo, cambiante e incierto necesita cada vez más de competencias más centradas en formar a docentes como investigadores de su propia práctica, comprometidos con el aprendizaje y desarrollo de los estu- 
diantes. Hoy por hoy, se necesita un profesorado que presente unas cualidades o competencias agrupadas en tres competencias profesionales básicas que como señala Pérez Gómez (2010), sustentan la mayoría de los programas innovadores de formación de docentes:

- Competencia para planificar, desarrollar y evaluar la enseñanza que pretende fomentar el desarrollo de las cualidades humanas deseables en los estudiantes.

- Competencia para crear y mantener escenarios abiertos, flexibles, democráticos y ricos culturalmente, donde se estimule un clima positivo de aprendizaje.

- Competencia para promover el propio desarrollo profesional y la formación de comunidades de aprendizaje con los colegas y el resto de agentes implicados en la educación.

Ahora bien, para el desarrollo de todas estas competencias es necesario asumir la competencia autoevaluativa como garantía de mejora y de reconocimiento de los propios errores para facilitar el crecimiento del docente como profesional (Méndez y Conde, 2018).

Para ello, los docentes han de entender la autoevaluación como el modelo que ofrece más posibilidades, compromiso, información, etc. para ayudarles a detectar los puntos fuertes y débiles de la práctica y tomar decisiones sobre ella con la intención de mejorarla. Como señalan los autores Airasian y Gullickson (1998, p.68.), "la autoevaluación del profesorado es un proceso en el que los docentes formulan opiniones valorativas sobre la adecuación y efectividad de su propio conocimiento, actuación, principios o consecuencias con el fin de mejorarse a sí mismos" Además, como comenta McLaughlin (1991), la autoevaluación puede estimular la motivación para el cambio más que ningún otro sistema de evaluación, puesto que son los mismos profesores los que analizan y valoran los aspectos débiles descubriendo orientaciones para la mejora. Especialmente para la profesora Rodríguez Diez (1996), la autoevaluación de la práctica docente es el conjunto de acciones (información, interpretación y valoración) llevadas a cabo por los profesores de un centro/ciclo/etapa para valorar la calidad de los procesos educativos que se desarrollan en las aulas de cara a introducir las modificaciones e innovaciones necesarias.

Recuperar el valor genuino por la autoevaluación pasa desde nuestro punto de vista por el reconocimiento de ésta como: a) reflexión del profesor sobre sus éxitos y fracasos y basándose en esta reflexión modificar su forma de enseñar, de evaluar, etc.; b) evaluación del profesor hecha por y para él; c) herramienta apropiada para 
tener una percepción más fiel de su actuación en el aula; y d) instrumento para poder identificar cuáles son sus necesidades de formación y crear estrategias para satisfacerlas, mejorando con ello su ejercicio profesional (Calatayud, 2008b; 2004; 2002; 2000).

\section{DISEÑO DE LA INVESTIGACIÓN}

El objetivo general de esta investigación es analizar las percepciones del profesorado de la escuela pública española acerca de cuáles son las dificultades más arraigadas para la emergencia de la autoevaluación entre el profesorado de Educación Primaria. Desde la mirada del profesorado podemos comprender cuáles son las dificultades para así potenciar una formación en autoevaluación que incida en que ésta sea entendida como una necesidad y una exigencia en la profesión docente hoy.

\subsection{Población}

Han participado en este estudio 150 profesores y profesoras de Educación Primaria de la Escuela Pública de España.

\subsection{Instrumento}

Se diseñó un cuestionario abierto para indagar cuestiones relativas a las dificultades que conllevan las prácticas autoevaluativas. En este artículo sólo se va incidir en una de las diez preguntas cualitativas que se plantearon sobre la autoevaluación docente como estrategia evaluativa y formativa de la práctica. El cuestionario fue previamente validado por un grupo de expertos (inspección educativa, asesores de formación del profesorado y profesorado universitario de la Universidad de Valencia). Se confirmó que las preguntas-tema eran adecuadas para explorar algunas ideas relativas a la autoevaluación como recurso para la formación docente.

A continuación se describen las valoraciones más destacadas por los participantes que han intervenido en este estudio. 


\section{DISCUSIÓN: LA VOZ DEL PROFESORADO}

Después de analizar las respuestas de la muestra participante en este estudio, se agruparon de forma sintética los comentarios más relevantes de los docentes a la pregunta planteada: ¿Cuáles son las dificultades más arraigadas para la propuesta de la autoevaluación como estrategia evaluativa y de mejora de la práctica docente?

Para el tratamiento de los datos se hizo un análisis cualitativo, organizando la información obtenida en las distintas categorías establecidas.

A continuación, se describen algunas de las categorías (dificultades) más importantes que aún están bastante arraigadas en los claustros de profesores de los Centros de Educación Infantil y Educación Primaria en España.

\subsection{La existencia de una nebulosa-atmósfera de trabajo en los equipos de profesores que dificultan los procesos de autoevaluación.}

Con el término nebulosa-atmósfera nos referimos a determinados factores que están condicionando una visión negativa hacia la autoevaluación. De entre ellos, el profesorado encuestado incidió, por ejemplo, en los siguientes:

a) La rutinización del trabajo docente.

Aunque se haya recibido formación en relación con los procesos de autoevaluación, el profesorado, muchas veces, está instalado en la comodidad, rutina, etc. y no quiere plantear ningún cambio porque éste, posiblemente, le va a producir una alteración en su dinámica concreta de trabajo. Según Santos Guerra (2000, p. 75), "la rutina tiene dos vertientes que se complementan y se condicionan: una personal por la que cada profesor mecaniza sus prácticas e, incluso, sus actitudes y su pensamiento. Otra Institucional que posee elementos personales y estructurales. Las dos tienen mucho peso en la escuela". Rutinización que según este mismo autor tiene una explicación, basada esencialmente en dos aspectos:

\section{a.1) La fuerte balcanización}

Cuando los autores Fullan y Hargreaves (1997), inciden sobre la cultura balcanizada que suele existir más en los centros de Educación Secundaria, están haciendo referencia a la "existencia de grupos separados y, a veces, competidores, que procuran conseguir la mejor posición y la supremacía sobre el resto, como si de ciudades-estados independientes y débilmente relacionadas entre sí se tratara" (p.81). 
Indudablemente, esta forma de trabajar conlleva a que en los Departamentos Didácticos, claustros, ciclos, se instalen políticas de mala comunicación entre los docentes. La indiferencia y el sálvese quien pueda forman parte de esta cultura. Incluso es frecuente poner zancadillas al profesorado que no es de su propio grupo de colegas con el objeto de salir airosos y mantener el poder a costa de lo que sea. Como señala Ball (1987), se generan disputas, conflictos con la idea clara de defender su territorio y estatus, ante las posibles "amenazas aparentes" de los demás compañeros. En contraposición a la cultura balcanizada, los autores Fullan y Hargreaves (1997), proponen la llamada "profesionalidad interactiva" que consiste en potenciar la cultura de colaboración. Propuesta basada en la reflexión, en el análisis sobre el sentido del trabajo docente, y la manera en que se desarrolla. Como muy bien argumentan estos autores, el reto de la profesionalidad interactiva es el reto del perfeccionamiento continuo de la escuela.

Además, como señala Hargreaves (1991), existen otra serie de culturas distintas a las balcanizadas que también pueden estar dificultando los procesos de autoevaluación. Algunas de ellas son: a) el individualismo rayano en el retraimiento que es una forma de ser y de estar en la escuela que se puede caracterizar por la incomunicación entre el profesorado; b) el individualismo obligado que viene impuesto por la autoridad administrativa $y$, sobre todo, se da en centros que existe una dirección que no implica a los profesores en la toma de decisiones, donde existen horarios rígidos, etc.; c) el individualismo elegido, donde los docentes prefieren el aislamiento total y/o parcial y d) la colegialidad artificial en la que los profesores trabajan juntos para la realización de algunas actividades concretas y luego cada uno vuelve a su quehacer independiente en sus respectivas clases.

\section{a.2.) El carácter funcionarial de la profesión docente}

Cuántas veces hemos oído en los centros educativos la siguiente frase: "para qué quiero hacer esto o aquello si a final de mes, haga lo que haga, voy a cobrar lo mismo que mi compañero/a que no se forma, no innova, etc." . El carácter funcionarial de la profesión conlleva a que muchas veces los docentes se instalen en mentalidades de rutina, de estabilidad en su trabajo, de comodidad, etc. que potencian posiciones de anclaje en las que da igual cómo se desempeñe la profesión docente porque, haga lo que se haga, la remuneración va a ser la misma. Sencillamente así lo describe Santos Guerra (2000, p. 87), cuando argumenta que "el carácter funcionarial de muchos de los profesionales que trabajan en la escuela pública (necesario para dar estabilidad y persistencia a los proyectos) se convierte a veces en una trampa. Amparados en la garantía de la continuidad en el trabajo se haga como se haga y pase lo que pase, es más fácil entregarse a la rutina y a la pereza". 
En general, la mayoría de las veces estas formas de actuar se manifiestan, como he comentado anteriormente, por el claro imperio de determinadas "culturas latentes" en los claustros, ciclos, etc. Con "culturas latentes" nos referimos a una serie de características culturales que forman parte de la institución escolar, sin que, por su carácter oculto e implícito se reflejen en el organigrama ni en los documentos pedagógicos y organizativos y que dificultan en gran medida el desarrollo de las prácticas autoevaluativas. Se trata, por ejemplo, de la cultura del individualismo profesional, la competitividad, la simplificación de los procesos educativos, la obsesión por la inmediatez de los resultados, la cultura del mínimo esfuerzo, la cultura del funcionariado, la cultura del reino de taifas en cada aula, etc. Estas asunciones están desvirtuando y dificultando, más si cabe, el genuino principio de las prácticas autoevaluativas. Prácticas que reclaman que el profesorado se sensibilice por nuevos valores: frente al individualismo, la colaboración; frente a la dependencia, la autonomía; frente al aislamiento, la comunicación; frente a la dirección externa, la autorregulación y crítica colaborativa. Es decir, por postulados que potencien la esperanza en prácticas educativas de autoevaluación que impliquen mejora y desarrollo profesional (Calatayud, 2009a; 2009b; 2009c; 2008a).

\section{b) La organización y funcionamiento de algunos equipos de docentes que no estimulan la innovación y dificultan todo tipo de iniciativas personales}

Indudablemente la descoordinación, la falta de organización, etc. entre los propios Departamentos Didácticos, ciclos y entre profesores del mismo Dpto, ciclo, etc. engendra comportamientos que no potencian la innovación ni, por supuesto, actitudes de cooperación entre los profesionales que trabajan en ellos. Además, esa falta de organización dificulta el que existan espacios para la reflexión, el intercambio de experiencias, etc.

\section{c) Los temarios que hay que cumplir y los sistemas de evaluación vinculados a ellos}

Muchas veces el profesorado se siente atado de pies y manos porque tiene que cumplir un curriculum prescrito que dificulta el plantear una serie de innovaciones en el aula. Se sienten como tecnócratas que tienen que desarrollar unos programas, temarios, etc. que muchas veces suelen generar dinámicas de trabajo encorsetadas en el descontento y en la desilusión. 


\subsection{Por problemas derivados del alumnado}

En varias ocasiones, el número de alumnos, los problemas de aprendizaje, la disciplina, el desinterés, etc. de los estudiantes dificultan procesos de innovación relacionados con la evaluación.

\subsection{Falta de apoyos sociales que consideren positivamente la profesión docente}

En la mayoría de las situaciones docentes, el profesorado se siente sumido en la mayor apatía ante la innovación y desarrollo profesional porque percibe y "siente en sus carnes", un descenso en la consideración social de su función como profesor, una escasa colaboración familiar, un aumento progresivo de las exigencias / demandas al profesorado, etc. Aspectos que están condicionando que el profesor se encierre en su caparazón sumido por la desesperación, inseguridad, ansiedad, desconsideración, desinterés profesional y malestar docente.

Un cambio de conducta favorable a innovar exige establecer incentivos que refuercen las nuevas manifestaciones conductuales mediante motivaciones extrínsecas (reconocimiento de los demás, mayor sueldo, etc.) o intrínsecas (aumento de la satisfacción personal, sentimientos de reconocimiento, etc.).

\subsection{Desmotivación del profesorado hacia la autoevaluación}

Cada vez se oye más en los centros educativos y fuera de ellos que el profesorado, sobre todo, de Educación Secundaria está desmotivado. Al docente no se le mima y, muchas veces, se encuentra sólo y sin capacidad de reaccionar ante los cambios que se le avecinan. Como señala Santos Guerra (2000, p. 79), "el problema de la motivación de los profesores es intrincado porque conduce a un círculo vicioso: si no están motivados no se entregarán en cuerpo y alma a la profesión y no disfrutarán de sus dimensiones más reconfortables. A su vez, si el ejercicio de la profesión no les reporta gratificaciones personales, no se sentirán motivados...Si el profesorado está desmotivado será difícil que vaya más allá del cumplimiento formal de sus obligaciones administrativas: estar a la hora, asistir a las reuniones, acudir a las aulas, impartir docencia, poner las notas..." 
Muchas veces esa falta de motivación por innovar viene condicionada por muchos factores. Factores que en la mayoría de los casos vienen determinados porque el profesorado está desarrollando una profesión que no entraba en sus aspiraciones y/o expectativas iniciales. Se encuentra sumido en dicho trabajo, bien sea, por intereses espurios, o bien por motivos de otra índole.

Otro aspecto que me parece sumamente importante es aquel que tiene que ver con el estatus del docente. La profesión de maestro goza, como todos sabemos, de un reconocimiento social relativamente pobre. A pesar de tener el mismo título que diplomados, socialmente "queda mejor", decir que eres un empresario a decir que eres un profesor, aunque se trate realmente de títulos del mismo grado. En la investigación realizada por Zubieta y Susinos (1992, p. 261), se constata que el deterioro de la imagen social del docente constituye uno de los principales factores de su malestar: "Confirman este aspecto los resultados cuantitativos y cualitativos obtenidos. Indican que, a pesar de que las situaciones laborales de los distintos grupos de profesores son asimismo diferentes, existe un acuerdo generalizado entre los docentes en que su oficio no tiene el prestigio social de hace unos años y que, en cualquier caso, el reconocimiento social y administrativo (formación, remuneración económica, etc.) es escaso".

Del mismo modo, consideramos que otro de los factores que determinan el potenciar la cultura autoevaluativa es la vocación que tenga el docente por su profesión. Entre otras razones porque una persona con vocación y motivación por la profesión docente sentirá la necesidad de evaluar su propia práctica, así como también, de seguir formándose y perfeccionándose a lo largo de su vida profesional.

\subsection{Falta de una mayor autonomía en los equipos docentes para llevar a la práctica procesos de autoevaluación}

Como afirma Santos Guerra (2000), cada institución escolar se ve convertida en un engranaje del sistema y no en un elemento dinamizador de su propio curriculum. Dicho engranaje dificulta que la escuela tenga autonomía para decidir y para llevar a la práctica los procesos autoevaluativos. Además, los profesores actúan dentro de instituciones organizadas jerárquicamente por lo que resulta mínima su participación. El grado de autonomía que goce el docente, así como, los recursos para poder desarrollar su profesionalidad con libertad, serán aspectos que influyen desmesuradamente en su desarrollo profesional. 


\subsection{Reivindicaciones de determinadas condiciones de trabajo}

"Se habla diariamente de problemas relacionados con las continuas dificultades que ha de solventar el docente para desarrollar su trabajo en el aula, de la sistemática dejación que los responsables políticos practican respecto a las continuas demandas, del distanciamiento e incomprensión social que instala la actividad docente en una situación cada día más distante de los fines, actitudes y valores que la práctica social establece como prioritarias. La demanda de recursos, materiales y humanos, colma una parte de esa necesidad reivindicativa legitima y necesaria, que no es sino el signo de una frustración que anida en la mayoría de los docentes y que aflora progresivamente con la edad. Hay problemas latentes relacionados con expectativas personales que la realidad incumple." (Revenga, 2002, p. 71).

Si partimos de la idea que una formación basada en procesos de autoevaluación tiene que posibilitar en el docente el desarrollo profesional, también es cierto que, las condiciones profesionales del profesorado también han de mejorar. Junto con las actividades de formación que se planifiquen, hay que desarrollar una serie de iniciativas que conduzcan a la mejora de la consideración social de la función docente y de la percepción y autoconcepto del profesorado sobre su propia profesión. Como señala Delors (1996, p.30) "el maestro tiene la impresión de estar solo, no únicamente porque ejerce una actividad individual, sino debido a las expectativas que suscita la enseñanza y a las críticas, muchas veces injustas, de que es objeto. Ante todo, desea que se respete su dignidad".

Para que la formación recibida sobre procesos de evaluación luego sea aplicada al aula es necesario disponer de determinados recursos pedagógicos, personales, económicos, didácticos, etc. que puedan hacer realidad los cambios. Además, se ha de contar con la variable tiempo para la reflexión, de una estabilidad en las plantillas docentes para que se puedan desarrollar innovaciones educativas desde una dimensión colegiada.

El contexto del docente, también influye de forma trascendente en los procesos de autoevaluación. Entendemos como contexto el medio en el cual el docente desarrolla su tarea, el entorno, así como también, las relaciones de éste con el resto de profesionales que trabajan en el mismo centro o instituto, con los padres, los alumnos, la Administración Educativa, etc. Además, la dirección del centro educativo resulta ser un órgano fundamental en los procesos de cambio y mejora de la enseñanza, debido a su poder como líder, gestor y mediador. Numerosas 
investigaciones identifican a la función directiva como agente de innovación. Su papel no sólo consiste en impulsar y potenciar el cambio, sino también cambiar personalmente, en su manera de proceder (Tejada 1996; Gairín, 1995)

\subsection{Escasa tradición investigadora del profesorado (sólo investigan los teóricos)}

Como señala Santos Guerra (2000, p. 78), "si el motor del cambio se sitúa fuera de la escuela es fácil que se produzca una pereza institucional que deje la iniciativa a los agentes externos. "Ellos sabrán, ellos dirán". Esta tesis resulta desprofesionalizadora. Los que piensan están fuera de la escuela, lo que pasa en el centro es fruto de las elucubraciones de los expertos que no trabajan en él. Los verdaderos responsables de las decisiones de cambio son los que dictan las normas desde los despachos".

Por mucha formación que reciba el profesorado sobre evaluación si continúa pensando que las innovaciones han de venir determinadas desde la Administración Educativa de nada sirve la asistencia a cursos, seminarios, etc. El docente entra en la dinámica de la meritocracia de la enseñanza sin incidir en la reflexión sistemática y en la idea de que no es él quien tiene en sus manos la mejora de la práctica profesional.

La escasa tradición investigadora del profesorado también está incidiendo negativamente en que los docentes sean agentes de cambio e innovación. Asimismo, es relevante también destacar que, en algunas ocasiones, el profesorado que innova suele ser mal visto por los demás compañeros-colega. Es lo que el autor Santos Guerra (2000), denomina "fagocitosis del innovador". Este fenómeno se suele observar cuando un docente realiza una innovación y los demás compañeros suelen verlo como una amenaza porque les obliga también a ellos a "ponerse las pilas". Los colegas suelen reaccionar descalificando a quien formula o realiza la innovación.

Además, como consecuencia de esa escasa tradición investigadora, en algunas ocasiones, el profesorado ante los fracasos y experiencias negativas opta por continuar haciendo lo que estaba desarrollando sin correr ningún riesgo, ni conflicto con el resto de los compañeros. Fracasos que conducen al escepticismo, inmovilismo, pereza y miedo. 


\subsection{Miedo a la incertidumbre y a los cambios que pueden provocar las prácticas autoevaluativas}

Muchas veces profesorado formado y preparado para realizar determinadas innovaciones educativas en relación con la evaluación tiene miedo e incertidumbre. La falta de confianza, la idea de que los verdaderos responsables de los cambios se sitúan fuera de la escuela, etc. determina un sentimiento de inseguridad, miedo a la novedad, a los cambios que suele provocar entre el profesorado la tendencia a continuar con sus rutinas y tradiciones. Como señala Morrish (1978) existen determinados factores que están incidiendo negativamente en el cambio de las escuelas. Factores que clasifica en tres grandes categorías:

Factores input, que proceden del exterior, como pueden ser: las resistencias al cambio procedentes del entorno, la ausencia de agentes externos que sirvan de estimulo, la actitud defensiva que muchos profesores profesan en relación con los cambios que no están dispuestos a introducir en sus prácticas, por determinados motivos: miedo, falta de recursos, etc.

Factores output, que proceden del interior, como pueden ser: la falta de incentivación a los docentes que innovan (escasas expectativas de promoción profesional), formación recibida insuficiente, inexistencia de un diagnóstico adecuado de las necesidades y demandas formativas del profesorado, etc.

Factores throughput o de tránsito que tienen que ver con aspectos relativos a la falta de apoyo por parte de los compañeros/as en el propio departamento, centro (resistencias a trabajar en equipo), mala organización y funcionamiento de los centros educativos, que no estimulan la innovación y dificultan todo tipo de iniciativas personales, escasez de recursos o medios para su implantación, etc.

De Miguel (1991), en su investigación titulada: "Estudio sobre el desarrollo profesional de los docentes no universitarios. Análisis de las resistencias a la innovación educativa", señala que las principales resistencias a la innovación educativa son las siguientes: no se tienen las condiciones necesarias para que se puedan implantar en los centros procesos de innovación. La necesidad de transformar las condiciones de trabajo en el aula y en los centros como requisito para propiciar estrategias de cambio. Además de estas resistencias se destacan las siguientes: la inseguridad e incertidumbre que ocasiona el cambio, falta de dominio de las herramientas necesarias, resistencia a que otros colegas (compañeros, alumnos, padres, etc.) participen en la resolución de problemas que los docentes tienen que solucionar diariamente. 


\section{UNA RESPUESTA: LA NECESIDAD DE FORMAR EN AUTOEVALUACIÓN}

Para iniciar con éxito prácticas autoevaluativas innovadoras, necesariamente, hay que poner en marcha una serie de fases:

Primera fase. La identificación del problema. Es en esta primera etapa dónde hay que detectar aquellos aspectos de la práctica docente que más producen incomodidad, curiosidad, deseo de cambiar al profesorado. La pregunta clave, quizás sea: ¿qué se va a evaluar? Cómo se puede suponer el éxito de esta primera fase reside en la autorrevisión de la práctica docente de forma rigurosa e intentando ser lo más objetivo posible. Se puede barajar la idea de colaboración de un experto o facilitador externo en dicho proceso. Indudablemente, la respuesta a la pregunta sobre ¿qué se va a evaluar? reside, necesariamente, en "indagar aquello que realmente se quiera mejorar y que valga la pena ser investigado.

\section{La segunda fase versaría sobre la obtención de información sobre el área o} dimensión de la práctica objeto de estudio. La pregunta a la que se debería dar respuesta sería: ¿qué información se va a recoger?

Se trata de tomar como referente el máximo de evidencias que ayuden a comprender y analizar el objeto de estudio elegido. Las fuentes de información, como es obvio, pueden y deben de ser variadas con el objetivo de obtener diferentes perspectivas del objeto evaluado. En general, los instrumentos que se pueden utilizar para acceder al objeto de estudio, se pueden clasificar en cinco bloques:

a) Herramientas de autorreflexión elaboradas por el profesor.

b) Actividades de retroalimentación del alumnado.

c) Los datos recogidos a través de la evaluación que se realiza a los estudiantes y la revisión de evidencias documentales.

d) Informaciones basadas en grabaciones de vídeo.

e) La participación de un observador externo.

Véase ahora la descripción de estos 5 tipos de estrategias:

\section{a) Herramientas de autorreflexión elaboradas por el profesor}

Se trata de actividades que el docente puede diseñar con el objeto de evaluar y comprender aquello sobre lo que se está investigando. Se podrían elaborar cuestionarios, listas de control, escalas de estimación, protocolos para recoger la información de la entrevista con el equipo directivo, protocolos para el análisis documental de 
las programaciones y de las actas de las reuniones, etc. A menudo la simple tarea de responder y de puntuarse a uno mismo puede llevar a la reflexión sobre lo que uno conoce y hace realmente. Así como también, sobre lo que se necesita mayor conocimiento o habilidad para poder actuar tan bien como a uno le gustaría.

Otro de los instrumentos utilizados para analizar la práctica docente puede ser el diario del profesor. La reflexión sobre el diario nos permitirá detectar problemas, hacer explícitas las concepciones y posibilitar mejoras. Desde nuestro punto de vista, el diario es uno de los instrumentos más útiles para llegar al conocimiento, análisis, comprensión y valoración de la realidad escolar. Al centrarse en la descripción de la dinámica del aula a través del relato sistemático y pormenorizado de los distintos acontecimientos y situaciones cotidianas ofrece, sin lugar a dudas, una panorámica general y significativa de lo que sucede en el aula. El diario según Porlan (1991), debe reunir una serie de características:

- No caer en la simplicidad de registrar sólo lo anecdótico y lo superficial, sino atender a un nivel más profundo de descripción de la dinámica del aula a través del relato sistemático.

- Debe de hacer referencia a la descripción del hecho, conducta, al análisis, a la valoración, a la reflexión (cuestionamiento de las teorías implícitas) y a las soluciones o alternativas (intervenciones).

\section{b) Actividades de retroalimentación del alumnado}

Ésta es una de las herramientas más importantes que el profesorado puede utilizar para evaluar el funcionamiento de las clases, la motivación e interés, la comprensión de lo que explica, asi como si la metodología utilizada conecta con los intereses de los estudiantes, etc. (Calatayud, 2008a).

c) Los datos recogidos a través de la evaluación que se realiza a los estudiantes y la revisión de evidencias documentales.

En este apartado se incluyen las producciones de los estudiantes, sus diarios, los resultados de los exámenes, etc. aspectos todos ellos que pueden ayudar al profesorado a evaluar su práctica docente. En cuanto a la revisión de evidencias documentales se destacan, entre otras, la planificación y la programación didáctica, etc. Estos instrumentos nos pueden ayudar en todo momento a valorar la dinámica de clase, así como a investigar y a reconducir muchos de los planteamientos curriculares (proyectos curriculares de centro, programación general anual, etc.). 
d) Informaciones basadas en grabaciones de vídeo. Se parte de la idea de que la autoevaluación debe de servir para mejorar el conocimiento que el docente tiene de su propia actuación.

e) La participación de un observador externo que ayude a superar, como plantea Solabarrieta (1996), el desfase entre la propia percepción del docente y la realidad de su actuación vista por otra persona.

En general, las posibles técnicas o estrategias más comunes que pueden ser utilizadas para profundizar en las prácticas autoevaluativas, siguiendo las orientaciones de Iwanicki y McEachern (1984), son las siguientes:

a) Las autoevaluaciones individuales, que no suelen precisar la colaboración de otra persona. Es decir, son aquellas que realizan los propios docentes a partir de cuestionarios de autoevaluación.

b) La autoevaluación con feed back, donde otros compañeros, alumnos, expertos o facilitadores externos ofrecen una retroalimentación al profesor.

c) Las autoevaluaciones interactivas, con procesos muy sistematizados de análisis compartidos con otras personas.

La tercera fase incide en la reflexión, toma de decisiones y elaboración del Plan de Mejora. Después de reflexionar sobre la información que se ha ido recogiendo e interpretando se ha de tomar una decisión: elaborar el Plan de Mejora. En esta fase se trata de contestar a la siguiente pregunta: ¿qué significado tiene esta información para mi práctica docente y para la mejora de la misma?

La cuarta fase incide en la aplicación del plan. En esta etapa es cuando, verdaderamente, se tiene que poner en práctica el Plan de Mejora. La pregunta a responder sería: ¿qué proceso debemos seguir para ejecutar el Plan de Mejora?

\section{La quinta fase consiste en la metaevaluación del plan o propuesta de acción} desarrollada. Se trata de someter a una nueva revisión el plan realizado, con el objeto de reflexionar si realmente se han producido los cambios oportunos y previstos.

A través de estas distintas fases que componen el proceso autoevaluativo se promueve la reflexión que conlleva una mejora de la práctica docente que incide en el desarrollo profesional del profesor. 


\section{A MODO DE CONCLUSIÓN}

A lo largo de este artículo se ha pretendido ofrecer la oportunidad al profesorado de reflexionar sobre el valor positivo que en sí mismo encierra la autoevaluación como "proceso facilitador" para evaluar la práctica docente y como recurso formativo. Dirigir nuestra mirada hacia prácticas autoevaluativas conlleva la idea de la evaluación como ayuda, crecimiento y desarrollo personal y profesional.

Desde esta premisa, a lo largo del estudio, se ha incidido en la idea de apostar por la autoevaluación:

- Como recurso de investigación y de formación porque ella es en si misma una forma de aprendizaje.

- Que está al servicio de quien enseña y de quien aprende para hacerla más reflexiva y para hacer de ella un instrumento de mejora.

Las aportaciones realizadas por 150 participantes en esta investigación indican el camino que se ha de seguir, ahora es cuestión de ir caminando y potenciando la relevancia de la autoevaluación como recurso formativo y evaluativo de la práctica docente. En este sentido y como decía Sócrates "nunca podemos vivir mejor que cuando nos esforzamos por mejorar". Y esa mejora pasa por entender y practicar una competencia autoevaluativa que forme al docente como profesional de la educación y le ayude a mejorar su práctica docente. La profesionalidad y las exigencias de una mayor calidad de la enseñanza nos impulsarán a ir caminando hacia la puesta en práctica de la competencia evaluativa. Competencia que ayuda a ser autocríticos, a mejorar, a reconocer los errores y a ayudar a crecer al docente como profesional. Pues bien, ante esta realidad sólo queda por cuestionarnos: ¿a qué estamos esperando?

\section{BIBLIOGRAFÍA}

Airasian, P. y Gullickson, A. (1998). Herramientas de autoevaluación del profesorado. Bilbao: Ediciones Mensajero.

Ball, S. (1987). "Staff during the teachers' industrial action: context, conflict and proletarianization". Bristish Journal of Sociology of Education. Núm 9 (3).

Bozu, Z. y Canto, P. (2009). "El profesorado en la sociedad del conocimiento: competencias profesionales docentes". Revista de Formación e Innovación Educativa Universitaria .Vol. 2. Núm 2.pp. 87-97. 
Calatayud Salom, A. (2000). "La Evaluación en la Educación Secundaria Obligatoria. Dificultades percibidas y estrategias de superación." Revista: Ciencias de la Educación, 2000. Núm 183. pp. 138-150.

Calatayud Salom, A. (2002). "La cultura autoevaluativa, piedra filosofal de la calidad en educación". Revista: Educadores. Núm 204.pp.357-375.

Calatayud Salom, A. (2004). "La autoevaluación de la práctica docente: una aventura plagada de dificultades y satisfacciones". Revista: Ciencias de la Educación. Núm 198-199. pp 151-171.

Calatayud Salom, A. (2007). "La evaluación como instrumento de aprendizaje y mejora. Una luz al fondo". En: A. Calatayud (Coord). La evaluación como instrumento de aprendizaje. Estrategias y técnicas. Madrid: MEC.

Calatayud Salom, A. (2008a). La escuela del futuro. Hacia nuevos escenarios. Madrid: CCS.

Calatayud Salom, A. (2008b). "Establecer la cultura de la autoevaluación". Revista: Padres y Maestros. Núm 314. pp 30-35

Calatayud Salom, A. (2009a). "Aprender de la evaluación para mejorar la práctica docente". Novedades Educativas. Núm 221.pp 42-45.

Calatayud Salom, A. (2009b). "Evaluar al profesorado. Un tema controvertido". Revista: Padres y Maestros. Núm 327.pp 23-27.

Calatayud Salom, A. (2009c). Entresijos de los centros escolares. Desvelarlos para mejorarlos. Málaga: Aljibe.

Cano E. (2005). Cómo mejorar las competencias de los docentes. Barcelona: Graó.

Cano E. (2008). "La evaluación por competencias en la educación superior." Revista de Currículum y Formación del profesorado, 12, 3, pp 1-15.

Coll, C. (2007). "Las competencias en la educación escolar: algo más que una moda y mucho menos que un remedio". Aula de Innovación Educativa. Núm 161. Pp 38- 46.

Charlier, E. (2005). Cómo formar maestros profesionales. Por una formación continua vinculada con la práctica. En: La formación profesional del maestro, estrategias y competencias. México: Fondo de cultura económica.

De Miguel, A. (1991). Estudio sobre el desarrollo profesional de los docentes no universitarios. Análisis de las resistencias a la innovación educativa. Madrid: CIDE.

Delors, J. (1996). La educación encierra un tesoro. Madrid: Santillana. Ediciones UNESCO.

Fullan, M. y Hargreaves, A. (1997). ¿Hay algo por que merezca la pena luchar en la escuela? Sevilla: M.C.E.P.

Gairín, J .(1995). Estudio de las necesidades de formación de los equipos directivos de los centros educativos. Madrid: CIDE. 
Guzmán, I. y Marín, R. (2011). "La competencia y las competencias docentes: reflexiones sobre el concepto y la evaluación". Revista Electrónica Interuniversitaria de Formación del Profesorado, 14 (1).pp 151- 163.

Hargreaves, A. (1991). Cultures of Teaching. Paper presented at the annual meeting of the A.E.R.A: Chicago.

Iwanicki, E.F. y Mceachern, L. (1984). "Using Teacher self-assessment to identify staff development needs". Journal of Teacher Education, 35 (2). pp 38-42.

Mclaughlin, M. W. (1991). Enabling Professional Development: What have we learned? En: A.Lieberman \& L. Millar (Eds) (1991). Staff Development for Education in the '90s. London: Teachers College Press.

Méndez, J.M. y Cobde, S. (2018). “La autorreflexión inicial: una estrategia para la mejora de la práctica docente". Revista Electrónica Interuniversitaria de Formación del Profesorado, 21 (1), pp. 17-31.

Morrish, I. (1978). Innovación y cambio en la enseñanza. Madrid: Anaya.

Pérez Gómez, A. (2010). "Aprender a educar. Nuevos desafíos para la formación de docentes." Revista Interuniversitaria de Formación del Profesorado, 68. Pp 37-60.

Perrenoud, PH. (2007). Diez nuevas competencias para enseñar. Barcelona: Graó.

Porlan, R. (1991). El diario del profesor. Un recurso para la investigación en el aula. Sevilla: Diada.

Revenga, A. (2002). Investigación-acción en la enseñanza secundaria. En: F. Imbernon. (2002), La investigación educativa como herramienta de formación del profesorado. Barcelona: Graó.

Rodríguez Diez, B. (1996). Hacia un modelo de autoevaluación de la práctica docente. En: AA. WV Dirección participativa y evaluación de centros docentes. Bilbao: Universidad de Deusto.

Santos Guerra, M.A. (2000). La escuela que aprende. Madrid: Morata.

Solabarrieta, J. (1996). Modelos de autoevaluación del profesor. En: AA. VV. Evaluación educativa. Evaluación Institucional. Instituto Universitario de Ciencias de la Educación: Salamanca.

Tejada, J. (1996). Liderazgo formal y procesos de innovación. En: M. A Zabalza. (Ed), Reforma Educativa y Organización Escolar. Santiago de Compostela: Tórculo.

Zubieta, J. C. y Susinos, T. (1992). Las satisfacciones e insatisfacciones de los enseñantes. Madrid: CIDE. 